\title{
A comparison of two techniques for reducing context-dependent forgetting
}

\author{
STEVEN M. SMITH \\ Texas A\&M University, College Station, Texas
}

\begin{abstract}
Recall is poorer when tested in a new environment than when tested in the original learning context. Two techniques for reducing this context-dependent forgetting were compared. One technique involved instructing subjects to recall their learning room(s), and the other attempted to establish multiple environmental retrieval cues by presenting lists in multiple rooms rather than all in the same room. Subjects were given three word lists to study in one or three rooms. All subjects were given a free-recall test in a new room, and half were asked to use remembered environmental context (EC) information to facilitate word memory. Multiple input contexts benefited only subjects who were uninstructed in the use of EC cues. Subjects given EC-recall instructions, however, recalled somewhat less in the three-room condition than in the one-room condition. The facilitative effects of the two techniques were not additive: EC-recall instructions benefited only one-room subjects. The results suggest that both EC-recall instructions and multiple learning contexts induce subjects to use contextual retrieval cues that are otherwise not spontaneously utilized, and that the greater the number of context cues stored in memory, the less accessible those cues become.
\end{abstract}

Context-dependent memory (CDM) has referred typically to the common finding that recall tested in a new context is poorer than recall tested in the original learning context (e.g., Smith, Glenberg, \& Bjork, 1978). Although CDM has been demonstrated with "context" defined in a variety of ways, referring, for example, to drug states (e.g., Eich, 1980), mood states (e.g., Bartlett \& Santrock, 1979), or postures (Rand \& Wapner, 1967), the present study is concerned with memory's dependence upon one's physical background surroundings, also known as environmental context (EC). The present study compared two techniques that had been shown to nullify the forgetting caused by testing memory in a new EC: the context-recall technique (Smith, 1979) and the multiple-learning-context technique (Smith, 1982).

The context-recall technique involves giving an instruction to subjects to generate their own environmental cues from memory, using remembered context rather than physically reinstated context cues. Using this EC-recall technique, subjects in Smith's (1979) study who were tested in a new room recalled as many list words as subjects tested in their original learning room. Those subjects tested in a new room

This research was funded by an expense grant from the Liberal Arts College at Texas A\&M University. Thanks are extended to Richard Mache for conducting the study and to Art Glenberg for his helpful comments in the development of the paper. Thanks are also due Bob Bjork and anonymous reviewers for suggestions regarding an earlier version of this paper. Requests for reprints should be sent to Steven M. Smith, Department of Psychology, Texas A\&M University, College Station, TX 77843. who were not given EC-recall instructions showed typical EC-dependent forgetting, recalling only twothirds the number of words recalled by the other groups. The EC-recall strategy remedied contextrelated forgetting, however, only when the learning EC was not difficult to remember.

The second technique used to nullify CDM was to present subsets of the learning materials in multiple contexts, rather than presenting them all in the same EC (Smith, 1982). On a free-recall test given in a new room, subjects who had multiple study rooms recalled more words and clustered more by sublists than subjects given a single study room. Recall did not differ among multiple-learning-room subjects whether those subjects were tested in a learning context (same-context, or SC, condition) or in a new context (different-context, or DC, condition), whereas the one-room SC subjects recalled $42 \%$ more than the one-room DC subjects. These results led Smith (1982) to conclude that the multiple-learning-room procedure improved recall of DC subjects and "immunized" learned material against the deleterious effects of EC change.

To explain the facilitative effects of multiple learning environments, at least two factors must be considered: the multiplicity of contextual cues, and the likelihood that subjects will use EC cues in recalling list words. Increases in either factor should improve memory, multiple learning rooms could potentially affect both factors.

A number of different lines of research endorse the notion that increasing the number of retrieval cues increases one's recall performance. Cue-overload 
theory (Watkins \& Watkins, 1975), for example, states that "the efficiency of a functional retrieval cue in effecting recall of an item declines as the number of items it subsumes increases"' (p. 443). Given a fixed set of items to recall, this principle suggests that increasing the number of retrieval cues should increase the effectiveness of each cue. Increasing the number of categories or subjective organizations has been found to improve recall performance in a number of studies (e.g., Mandler \& Pearlstone, 1966; Tulving \& Pearlstone, 1966).

In another related area, a modification of encoding variability theory used to explain the lag effect in free recall (Glenberg, 1977) states that "an item repeated after a long lag is associated with more different groups than an item repeated after a short lag. These multiple retrieval routes give the item repeated after a long lag an advantage in retrieval, and hence in recall" (p. 283). The multiple retrieval routes are an adaptive mechanism: Given that some retrieval routes are forgotten, it is to the subject's advantage to have multiple retrieval routes rather than a single route. In the case of EC cues, it may be that, because some $\mathrm{EC}$ information is forgotten, establishing multiple EC cues ensures that some EC retrieval cues will be memorable at the time of recall.

A different explanation of the multiple-learningroom enhancement of recall focuses not upon the multiplicity of environmental cues, but rather upon the likelihood that subjects will use EC cues. Smith (1979) found that simply instructing subjects to use self-generated EC cues improved recall of DC subjects by $50 \%$; apparently, many subjects do not spontaneously use the EC-recall strategy when learning occurs in one room. It may be, however, that moving subjects from room to room during learning calls attention to EC information. If environmental information is given extra attention, it may become an important enough component of episodic memory that subjects spontaneously recall EC cues during free recall. Essentially, this explanation states that multiple learning rooms and ECrecall instructions function the same way, by inducing subjects to use remembered EC information to cue memory of list words.

According to the idea that multiple learning rooms simply induce subjects to recall EC cues, an ECrecall instruction should improve memory for a onelearning-room condition more than it would for a multiple-room condition, since the multiple-room subjects would be spontaneously using recalled EC information. Furthermore, a one-room group given EC-recall instructions should recall the same number of words as a multiple-room group.

The idea that EC-cue multiplicity improves recall predicts that multiple-room groups should recall more than one-room groups, and that instructions to recall EC cues should have an additive, rather than an interactive, effect with number of learning contexts.

The present experiment tested these predictions by presenting subjects with three sets of words, with each set being presented in a different room or else all in the same room. Free recall of all three lists was tested in a new context; half of the subjects were given EC-recall instructions prior to free recall of the words, and half were uninstructed with regard to the EC-recall technique. The subjects were also preexperimentally tested and classified as field dependent or field independent, since a previous study had suggested that field-dependent subjects may be more susceptible to context-dependent memory (Smith \& Rothkopf, in press).

\section{METHOD}

\section{Subjects}

Student volunteers from the introductory psychology classes at Texas A\&M University were presented with the Group Embedded Figures Test (GEFT; Oltman, Raskin, \& Witkin, 1971) and assigned to either the field-dependent or the field-independent recruitment list. Sixty subjects recruited from each list, a total of 120 volunteers, were assigned randomly to each of the four treatment conditions. Groups ranging in size from 4 to 6 subjects contained as even as possible a mix of both subject types. Each group participated for approximately $1 \mathrm{~h}$, with subjects fulfilling a course requirement with their participation.

\section{Procedure}

The subjects were asked to study three word lists presented by a male voice on audiotape. Words were presented at 3-sec intervals. Between lists, the subjects were asked to wait in the hall for $5 \mathrm{~min}$ and to refrain from discussing the experiment or the word lists during those times. In the one-room conditions, the subjects were asked to sit in the same seats for all three lists. After the 5-min break following the third list, they were taken to the test room (a new room) and were given taped recall instructions. Context-recall instructions asked the subjects to think about their learning environment(s), to list three objects seen in each of those rooms, and to use their memory of the learning rooms to help them recall list words from all three lists. The three-room group was asked to carry out these EC-recall instructions for all three rooms. Free recall followed the context-recall instructions for the EC-recall groups. Groups not given EC-recall instructions (uninstructed groups) were given the free-recall test without prior instructions. The taped free-recall instructions advised the subjects to write down words from all three lists in any order. They were given $5 \mathrm{~min}$ for the free-recall test.

\section{Design}

The three between-subjects variables were combined in a $2 \times 2 \times 2$ (instruction $\times$ number of rooms $\times$ GEFT) factorial design. The instruction variable consisted of EC-recall and uninstructed groups, GEFT was high (field independent) or low (field dependent), and number of rooms was one-room or three-rooms. In the three-room condition, all subjects heard List 1 in Room $A$, List $\mathbf{2}$ in Room B, and List $\mathbf{3}$ in Room C. For one-room subjects, one-third heard the list words in Room A, one-third in Room B, and one-third in Room C.

\footnotetext{
Materials

The GEFT consists of 25 embedded-figure problems, of which only the last 18 are scored; unknown to the subjects, the first 7 items are not scored and are treated as warm-up problems. Sections 2 and 3, each containing 9 problems, are presented to sub-
} 
jects for $5 \mathrm{~min}$ apiece. A perfect score is 18 correct. High scorers scored above 13 (the median of the pretested classes); low scores were those below 13. Each problem consists of a simple geometric form that is embedded in a complex visual context. The subject's task is to trace over one simple form in each complex figure.

Each of the three 32-word lists consisted of common four- and five-letter-one-syllable English nouns drawn from the Kučera and Francis (1967) word-frequency norms. Words were of a frequency of 25 per million or more.

\section{Environmental Contexts}

The four rooms were selected to be as different from each other as possible. Learning Room A was a small laboratory cubicle approximately $6 \times 6 \mathrm{ft}$ in size and contained a collapsible table that was folded against the wall, photographs and painting on the walls, and seven chairs crowded into the small space. Room B was a classroom with a large blackboard on one wall and windows overlooking the Texas A\&M campus on two sides; it seated 25 students. Room $\mathrm{C}$ was a long, narrow laboratory room with bare walls except for a mirror covering most of one wall, wooden cabinets, and rows of desk chairs for 10 people. The test room, Room D, was a small classroom with doors leading to other rooms in all four walls, chairs and black laboratory tables with seating for 20 people, pieces of laboratory equipment scattered about, and a green chalkboard that was rolled in front of the entrance used by the subjects.

\section{RESULTS}

Separate $2 \times 2 \times 2$ (instruction $\times$ number of rooms $\times$ GEFT) ANOVAs were computed for total number of words recalled and for proportion of clustering by list.

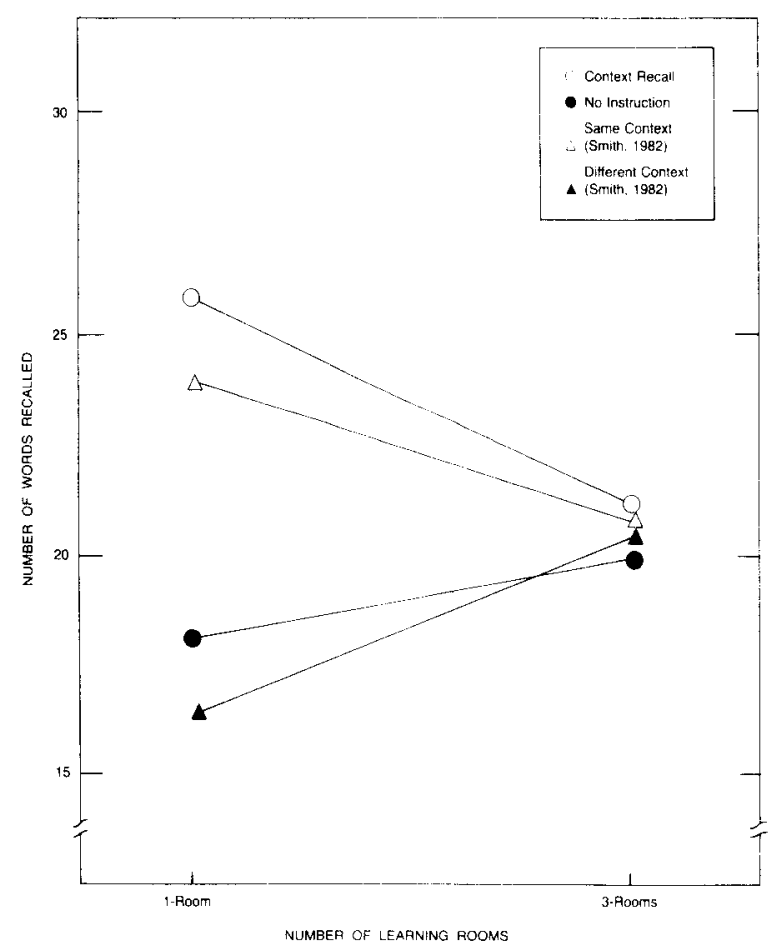

Figure 1. Mean number of words recalled in the present experiment and in Smith's (1982) study as a function of number of learn. ing rooms and of testing conditions. Maximum possible was 96 words.

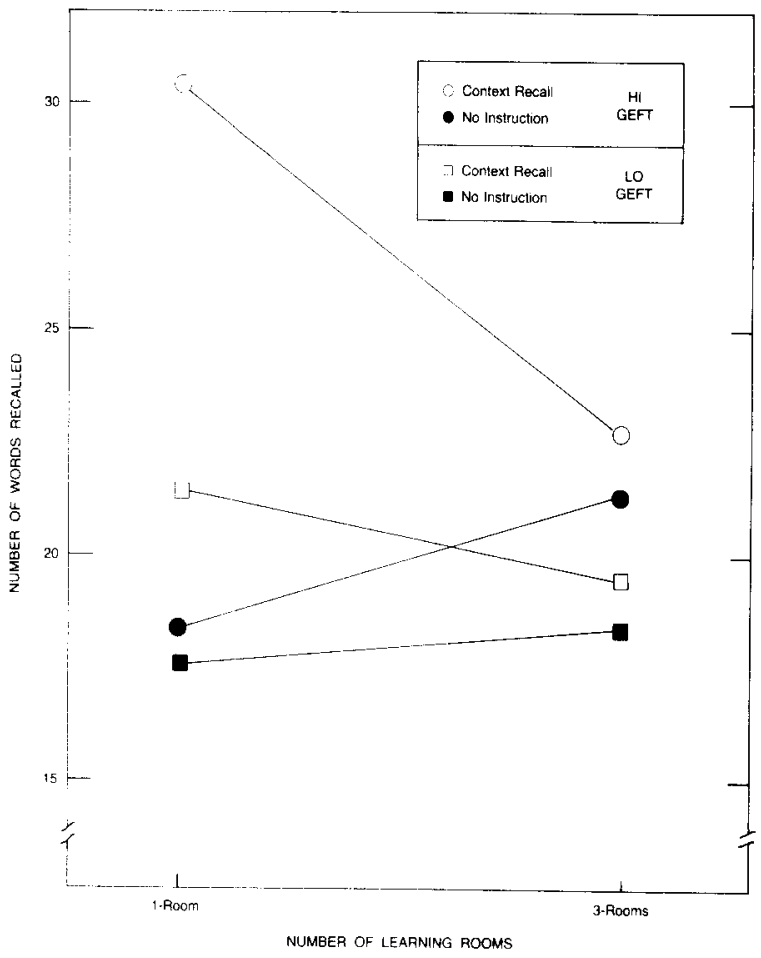

Figure 2. Mean number of words recalled as a function of number of learning rooms, recall instructions, and field dependence. Maximum possible was 96 words.

The analysis of the total number of words recalled per subject indicated that the context-recall groups recalled more than the uninstructed groups $[F(1,112)$ $=7.03, \mathrm{MSe}=88.98, \mathrm{p}<.01$ ] (see Figure 1). This is consistent with the results of Smith (1979). On the average, context-recall subjects recalled $21 \%$ more words from the three lists than did uninstructed subjects.

The facilitative effect of the context-recall instruction was greater for the one-room condition than for the three-room condition, as indicated by the instruction $\times$ number of rooms interaction $[F(1,112)=3.75$, $\mathrm{p}=.055$, although this result was only marginally significant. The EC-recall instruction improved recall by 8 words ( $44 \%$ of the uninstructed groups' mean) for the one-room groups, as compared with a mere 1.2 -word (6\% of the uninstructed groups' mean) improvement for the three-room conditions. NewmanKeuls analyses indicated a significant difference between the performance of the one-room-uninstructed group and the one-room/EC-recall group, whereas the three-room/EC-recall group's performance did not differ from that of the three-room/uninstructed group $(\alpha=.05)$. The instruction $\times$ GEFT interaction was not significant $[F(1,112)=1.44]$, although Newman-Keuls analyses $(\alpha=.05)$ indicated that the simple effect of instruction in the one-room condition was significant for field-independent subjects, but not for field-dependent subjects (see Figure 2). It 
should be noted that field-dependent and fieldindependent subjects showed the same effects, but the effects were greater for the field-independent individuals.

The instruction $\times$ number of rooms interaction; depicted in Figure 1, is compared with the results of Experiment 3 from Smith's (1982) study of multiple learning rooms. The simple effect of number of rooms for the EC-recall subjects in the present study is similar to the effect of number of rooms for SC subjects in Smith's (1982) study, and the simple effect of number of rooms for uninstructed subjects is similar to the effect of number of rooms for DC subjects in that study. To further examine the effects of number of rooms under both instruction conditions, separate $2 \times 2$ (number of rooms $\times$ GEFT) ANOVAs were computed for EC-recall and uninstructed subjects. Although previous studies had found facilitative effects of increasing the number of learning contexts for uninstructed DC subjects (Smith, 1982; Smith \& Rothkopf, in press), the trend in the same direction in the present experiment did not reach significance $[\mathrm{F}(1,56)=1.11, \mathrm{MSe}=52.10]$. For $\mathrm{EC}$ recall subjects, recall was worse in the three-room condition than in the one-room condition. This trend was not quite significant, however $[F(1,56)=2.63$, $\mathrm{MSe}=125.85, \mathrm{p}=.11]$.

A $2 \times 2 \times 2 \times 3$ (number of rooms $\times$ instruction $\times$ GEFT $\times$ list number) ANOVA was calculated using number of words recalled per list as a repeated dependent measure. This analysis revealed a trend suggesting a possible interaction between number of rooms $\times$ instruction $\times$ list number $[F(2,224)=2.82$, $\mathrm{MSe}=9.14, \mathrm{p}=.06 \mathrm{]}$. As can be seen in Figure 3, the effect of the EC-recall instruction, as well as the effects of multiple rooms, were more pronounced on Lists 2 and 3 than on List 1. For EC-recall subjects, the recall decrement caused by multiple rooms became more pronounced on each successive list, and for uninstructed subjects, the benefit of multiple learning contexts, absent in the recall of List 1, appeared only on Lists 2 and 3 . No other effects or interactions in this analysis approached significance.

Clustering scores for the various experimental conditions showed roughly the same pattern as the freerecall scores. Clustering scores were calculated by dividing the total number of adjacencies (i.e., words from the same list recalled consecutively) by the total number of possible adjacencies in the recalled words (i.e., total number of words recalled minus the number of lists from which at least one word was recalled). The ANOVA using clustering scores found a marginally significant effect for the number of rooms $x$ instruction interaction $[\mathrm{F}(1,112)=3.80, \mathrm{MSe}=1,774.24$, $\mathrm{p}=.054]$. As in the free-recall scores, the EC-recall instruction improved clustering for the one-room condition, but not for the three-room condition (see Figure 4). No other effects were significant.

\section{DISCUSSION}

The present results, together with those of prior studies (Smith, 1979, 1982), support the ideas that (1) incidentally stored EC information comprises potentially powerful retrieval cues, although such cues are not always utilized, (2) both multiple learning contexts and EC-recall instructions encourage subjects to utilize contextual cues to enhance recall, and (3) increasing the numbers of incidentally stored EC cues makes them more difficult to access and use as retrieval cues. Speculation that the beneficial effects of multiple learning contexts (for uninstructed DC subjects) and instructions to use self-generated context cues may be additive was clearly not supported, since a combination of the two techniques produced recall no better than that produced by either technique alone.

The conclusion that EC-recall instructions and

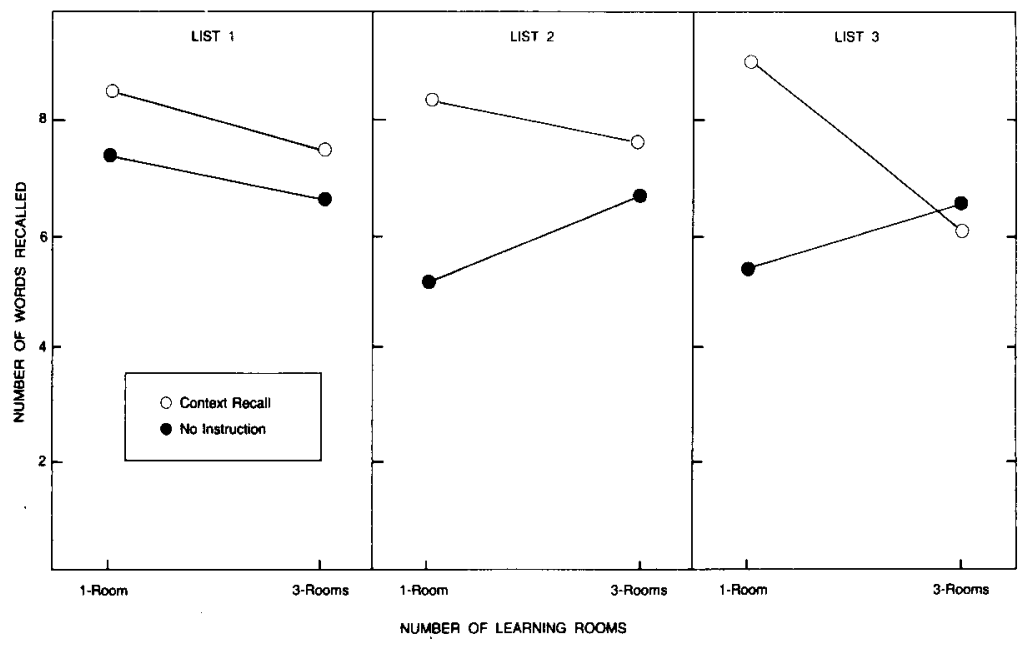

Figure 3. Mean number of words recalled per list as a function of number of learning rooms and of recall instructions. Maximum possible was 32 words per list. 


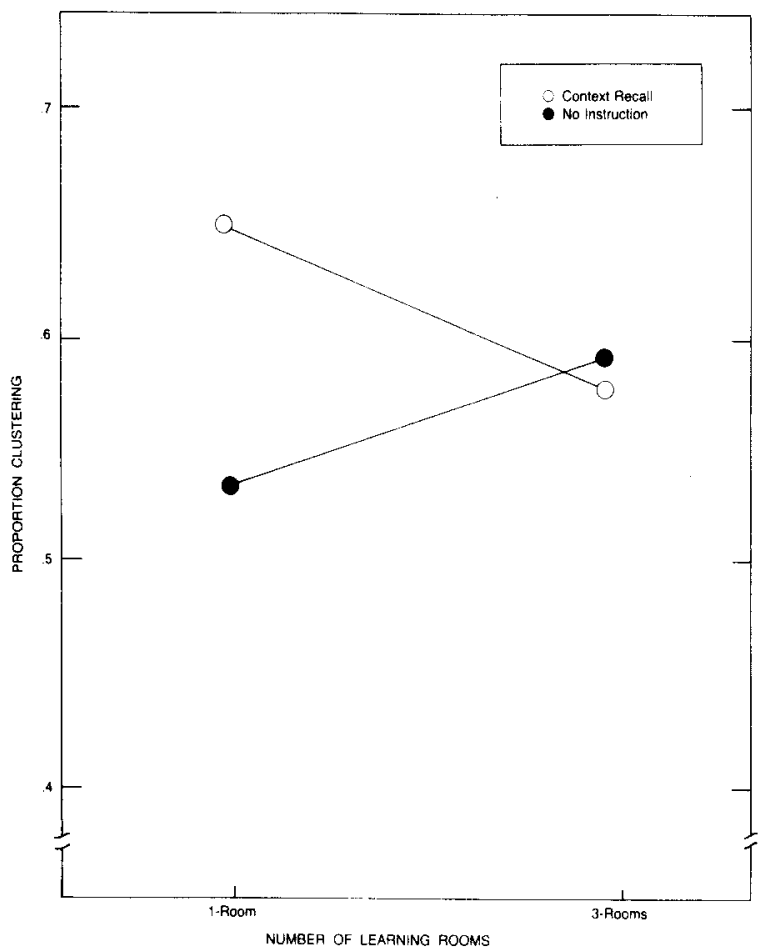

Figure 4. Mean clustering scores as a function of number of learning rooms and recall instructions. Maximum score possible was 1.0.

multiple learning contexts have identical effects would be an oversimplification of the results. Although there was no strong statistical support of the finding that recall was worse in the three-room instructed condition than in the one-room instructed condition, a trend in that direction appeared both in the present study and in the Smith (1982) study (in the one-room SC and three-room SC conditions; see Figure 1). This small, but consistent, trend could result from the combination of a facilitative effect (i.e., inducing subjects to utilize context cues) with a negative effect (i.e., reducing cue accessibility by increasing the number of cues). This interpretation is also in line with the finding that the benefit of multiple learning contexts for uninstructed subjects, although consistent, is small and, in some cases (including the present study), not statistically significant. The possibility that increased numbers of context cues reduce cue accessibility might explain why the benefit of multiple learning contexts is not more robust.

More evidence that may support this interpretation of the results is the marginally significant number of rooms $\times$ instructions $\times$ list number interaction. This interaction shows that the negative effect of multiple learning contexts in the instructed condition increased successively across lists (see Figure 4). Such an interaction is consistent with the idea that successively experienced ECs become less and less accessible, making them less useful as retrieval cues.
A number of mechanisms for forgetting caused by multiple learning contexts could be possible. For example, learning additional EC information could interfere with memory of list words, although the dissimiarlity between EC information and list words makes the possibility unlikely. Another possibility is that output interference makes each additional set of EC cues more difficult to access. Since most subjects in the present study began recalling List 1 words first, and because EC cue accessibility did not appear to suffer until Lists 2 and 3 were recalled, this output interference explanation seems reasonable. A related explanation could be that multiple contexts could interfere with each other, either proactively or retroactively. This last explanation was further supported by Smith's (1979, Experiment 3) study, which found that EC-recall instructions did not improve memory for subjects who had experienced five experimental rooms prior to their free-recall test.

Whether or not multiple EC cues decrease memory under conditions of physical EC reinstatement and self-generated EC cues, the multiplicity of context cues certainly did not improve memory in those conditions. The notion that memory performance improves simply because the number of retrieval routes increases is untenable. One must also consider the accessibility and utilization of these retrieval routes; cues are not useful unless they are both accessible and used. Other investigators have often pointed out the importance of cue accessibility. For example, Tulving and Pearlstone (1966) showed that word recall was greatly improved when category cues were provided, and Dong and Kintsch (1968) found improved recall when labels of subjective organizations were given. Glenberg (1977), in his discussion of encoding variability, limited the benefits of multiple retrieval routes to situations in which all of the subjective organizations in memory are equally accessible.

Although the present study failed to find a new way of nullifying context-dependent memory, it helped to eradicate two known methods, the use of multiple learning contexts and the use of self-generated context cues. Memory appears to benefit when EC cues are utilized, although inaccessibility of such cues diminishes their usefulness.

\section{REFERENCES}

Bartlett, J. C., \& Santrock, J. W. (1979). Affect-dependent episodic memory in young children. Child Development, 5, 513-518.

Dong, T., \& Kintsch, W. (1968). Subjective retrieval cues in free recall. Journal of Verbal Learning and Verbal Behavior, 7, 813-816.

Еich, J. E. (1980). The cue-dependent nature of state-dependent retrieval. Memory \& Cognition, 8, 157-173.

Glenderg, A. M. (1977). Influences of retrieval processes on the spacing effect in free recall. Journal of Experimental Psychology: Human Learning and Memory, 3, 282-294.

KuCEera, H., \& Francis, W. N. (1967). Computational analysis 
of present-day American English. Providence, RI: Brown University Press.

Mandler, G., \& Pearlstone, Z. (1966). Free and constrained concept learning and subsequent recall. Journal of Verbal Learning and Verbal Behavior, 5, 126-131.

Oltman, P. K., Raskin, E., \& Witkin, H. A. (1971). Group embedded figures test. Palo Alto, CA: Consulting Psychologists Press.

RAND, G., \& Wapner, S. (1967). Postural status as a factor in memory. Journal of Verbal Learning and Verbal Behavior, 6 , 268-271.

Sмiтh, S. M. (1979). Remembering in and out of context. Journal of Experimental Psychology: Human Learning and Memory, $5,460-471$.

SMitH, S. M. (1982). Enhancement of recall using multiple environmental contexts during learning. Memory \& Cognition, 10, 405-412.
Smith, S. M., Glenberg, A. M., \& Bjork, R. A. (1978). Environmental context and human memory. Memory \& Cognition, 6, 342-353.

Sмiтh, S. M., \& Rothkopf, E. Z. (in press). Contextual enrichment and distribution of practice in the classroom. Cognition and Instruction.

Tulving, E., \& Pearlstone, Z. (1966). Availability versus accessibility of information in memory for words. Journal of Verbal Learning and Verbal Behavior, 5, 381-391.

Watkins, O. C., \& Watkins, M. J. (1975). Buildup of proactive inhibition as a cue-overload effect. Journal of Experimental Psychology: Human Learning and Memory, 104, 442-452.

(Manuscript received January 1, 1984; revision accepted for publication May 2, 1984.) 